\title{
Surgical Treatment of Pulmonary Arterial Thrombosis
}

\author{
Edmo Atique Gabriel, Camila Alcalde Mazza and Marina Alves Jacintho de Mello* \\ Union of the Colleges of the Great Lakes- UNILAGO, Brazil \\ ${ }^{*}$ Corresponding Author: Marina Alves Jacintho de Mello, Union of the Colleges of the Great Lakes- UNILAGO, Brazil; E-mail: marinajmello@hotmail.com
}

Received: March 03, 2020; Accepted: March 07, 2020; Published: April 12, 2020

\section{Summary}

Right Thromboses Floating (RHT) are in transit from the legs to the pulmonary arteries and are therefore a severe form of Venous Thromboembolism (VTE) with a high early mortality rate without treatment. There is a lack of evidence-based recommendations for its management, which is why, motivated the accomplishment of this study, dealing with the subject, addressing the surgical treatment of pulmonary arterial thrombosis, considering professional experiences in the surgical management of the thrombus in transit and Pulmonary Embolism (PE).

Keywords: Pulmonary arterial thrombosis, Pulmonary embolism, Surgical treatment

\section{Introduction}

The presence of thrombi in right heart chambers may be a consequence of Venous Thromboembolism (VTE) or may develop in situ as a consequence of cardiac conditions. Transient Thrombus (TT) or floating thrombus is defined as a thrombus temporally located in the right cardiac chambers en route to the Pulmonary Artery (AP). It is, therefore, a severe manifestation of VTE and very often (more than 90\%) is associated with Deep Vein Thrombosis (DVT) or Pulmonary Embolism (PE). Given the high mortality rate without treatment (90\%) and being very early (in the first 24 hours), monitoring in critical units and urgent treatment (including systemic fibrinolysis or surgical embolectomy, in addition to conventional anticoagulation) is justified. TT is an uncommon manifestation of symptomatic PE, with an approximate frequency of $4 \%$. Currently, there is insufficient evidence on the best treatment option, and the recommendations are based on conclusions drawn from case series. The objective of this study is to discuss theoretical findings and experience of early surgical treatment of patients with TT and PE.

\section{Discussion on the Topic}

The presence of thrombi in the right cardiac chambers may be a consequence of VTE or may develop in situ as a consequence of cardiac conditions. This differentiation is of great importance considering that they have different treatment and prognosis [1]. TT or floating thrombus is defined as thrombus temporarily located in the right cardiac chambers en route to the PA. It is, therefore, a severe manifestation of VTE and with very high frequency (more than 90\%) is associated with DVT or PE [2]. Thus, the presence of a TT confirms the diagnosis of PE and implies the desire for immediate treatment without the need for additional diagnostic tests. Three types of right thrombus were described using the Transthoracic Echocardiogram (TTE): type A thrombi are the most common and tend to be large, free floating masses with a high propensity for distal embolization; Type B thrombi are small clots of immobile right chambers attached to walls originating in situ; finally, type $\mathrm{C}$ thrombi are rare and have great mobility mimicking atrial myxomas. TT is an uncommon manifestation of symptomatic PE, with an approximate frequency of $4 \%$.

Although coexisting Right Heart Thrombus (RHT) does not commonly occur in patients with acute symptomatic PE, studies have validated THR as a predictor of poor prognosis [3]. Despite anticoagulation, the mortality rate has been reported high (23.2$44.7 \%$ ), especially in the first 24 hours [3]. In a recent meta-analysis, which included 15,220 patients with symptomatic acute PE, patients with detectable RHT concomitantly with echocardiography had a three-fold increased risk of short-term death compared to patients without RHT [4]. Similar results were found in a study of patients with PE from the Computerized Registry of the Thrombotic Infirmary (RIETE), which included 12,441 patients with PE, of which 325 had RHT.

Severe hypoxemia and the occurrence of cardiac arrest were significantly related to in-hospital mortality in patients with concomitant acute and concurrent PH. For these reasons, early diagnosis and treatment are essential. However, some studies have shown that clinical prognostic scores (PESI, simplified PESI) have demonstrated excellent accuracy for the identification of patients with low risk of short-term complications and have shown that coexisting TT in normotensive patients with PE did not lead to greater all-cause mortality. However, the data suggest an increased risk of mortality for patients with TT and RV dysfunction. On the other hand, the prognosis is generally good after discharge.

Most TT is detected by TTE in patients diagnosed or suspected of acute PE. Therefore, TTE is an important tool for early recognition. In case of doubt, it would be necessary to perform a transesophageal echocardiogram, which is also useful in the detection of thrombi in PA and in the coexistence of Patent Foramen Ovale (FOP). The presence of FOP should be taken into account as it is a source of paradoxical embolism (emboli originating within the venous system, which passes through a FOP and enters the systemic circulation) and may be a 
site for the clot to be lodged. Some authors such as Barrios et al and Bodia favor the surgical removal of these thrombi with simultaneous pulmonary embolectomy due to the imminent risk of displacement, which can lead to massive PE or paradoxical systemic embolism. Despite its prognostic importance, current guidelines do not propose that echocardiography be performed routinely in all patients with acute PE or in all patients with a low-risk PESI assessment. However, they stimulate the evaluation of right ventricular function by echocardiography and / or measurement of cardiac biomarkers if, after clinical evaluation, there is uncertainty about whether patients need more intensive monitoring or thrombolytic therapy [4].

Current guidelines suggest that patients with acute hypotension (ie systolic BP $<90 \mathrm{mmHg}$ for $15 \mathrm{~min}$ ) and no high risk of bleeding should be treated with thrombolytic therapy. The development of hypotension suggests that thrombolytic therapy is indicated. Deterioration that has not resulted in hypotension may also lead to the use of thrombolytic therapy. For example, there may be a progressive increase in $\mathrm{HR}$, a decrease in systolic $\mathrm{BP}$ (which remains $>90 \mathrm{mmHg}$ ), increased jugular venous pressure, worsening of gas exchange, shock signals (eg, cold sweat, diuresis reduction, confusion ), progressive dysfunction of the right heart on echocardiography, or increase in cardiac biomarkers [4]. However, few studies, only series of cases and retrospective studies, have compared thrombolysis with surgical therapy in acute PE and have recommended considering surgical pulmonary embolectomy in case of hypotension and contraindication for thrombolysis or life-threatening situations such as thrombolysis failure, RV failure, cardiogenic shock and TT.

According to current guidelines, in patients with TT, the therapeutic benefits of thrombolysis remain controversial. Surgical embolectomy is justified in some cases of TT, for example, those with coexistence of FOP. The ideal treatment for concomitant acute and concurrent $\mathrm{PH}$ is not currently defined due to the absence of randomized clinical trials and should be considered on a case-by-case basis by multidisciplinary teams following a risk-benefit assessment [4]. Patients who did not receive therapy had a mortality rate of 90 to $100 \%$. In addition, the mortality rate is high $(21.1 \%)$ in the first 24 hours, which justifies an immediate treatment. Thus, inotropic support with catecholamine infusion should be prepared immediately after the diagnosis of TT and administered as soon as the patient's blood pressure drops below $100 \mathrm{~mm} \mathrm{Hg}$ or there is suspicion of cardiogenic shock. It may be preferable to admit patients to an ICU because sudden death is a risk and mechanical ventilation may also be required. Different therapeutic approaches have been reported for acute PE with concomitant TT: anticoagulation with Unfractionated Heparin (UFH), systemic thrombolysis with Recombinant Tissue Plasminogen Activator (RTPA), surgical embolectomy with exploration of the right chambers and pulmonary arteries in complete extracorporeal circulation, and endovascular thrombectomy.

According to Marti (2014), systemic thrombolysis and surgical embolectomy were more likely to survive $(81.5 \%$ and $70.45 \%$, respectively) than anticoagulation (47.7\%). However, there are contradictory data regarding the management of TT; a recent study that included patients with acute RI associated with TT from the RIETE registry showed that there were no significant differences in mortality and bleeding between reperfusion (thrombolysis, surgery) and anticoagulation therapy. Regarding the superiority of thrombolysis or surgical thrombectomy, [4] say that both are effective strategies, with a slight increase in the probability of survival with thrombolysis in some studies. They were, however, plagued by selection bias, small numbers and lack of comparable groups. A prospective, randomized, well-planned study is needed to determine the optimal treatment of acute PD and concomitant TT.

\section{Conclusion}

Transit thrombus is an uncommon and severe manifestation of VTE. The high rate of early mortality ensures rapid and effective treatment. Surgical embolectomy in patients with PE and concomitant thrombus in transit may be an effective treatment in selected patients, although the current evidence to support this approach is not definitive.

\section{References}

1. Arboine-Aguirre L, Figueroa-Calderón E, Ramírez-Rivera A (2017) Trombo em trânsito e tromboembolismo pulmonar submassivo tratado com sucesso com tenecteplase. Gac Med Mex 153: 129-133.

2. Athappan G, Sengodan P, Chacko P (2015) Eficácia comparativa de diferentes modalidades para tratamento de trombos cardíacos direitos em trânsito: uma análise conjunta. Vasc Med 20: 131-138.

3. Agarwal V, Nalluri N., Shariff MA (2014) Embolia grande em trânsito - um dilema terapêutico não resolvido (relato de caso e revisão da literatura). Coração Pulmão 43: $152-154$.

4. Barrios D, Rosa-Salazar V, Morillo R (2017) Significado prognóstico dos trombos do coração direito em pacientes com embolia pulmonar sintomática aguda: revisão sistemática e metanálise. Baú de 151: 409-416.

\section{Citation:}

Edmo Atique Gabriel, Camila Alcalde Mazza and Marina Alves Jacintho de Mello (2020) Surgical Treatment of Pulmonary Arterial Thrombosis. J Cardiol Clin Pract, Volume 3(1): 1-2. 